\title{
EXPERIMENTAL ASSESSMENT OF 3D NARROW SPACE MAPPING TECHNOLOGIES
}

\author{
G. Piniotis, S. Soile, F. Bourexis, M. Tsakiri*, C. Ioannidis
}

School of Rural and Surveying Engineering, National Technical University of Athens, Greece (piniotis@survey.ntua.gr, ssoile@survey.ntua.gr, fotis.bourexis@gmail.com,mtsakiri@central.ntua.gr, cioannid@survey.ntua.gr)

\section{Commission II, WG II/2}

KEY WORDS: SLAM, LiDAR, image-based mapping, point cloud, narrow space, sensors evaluation

\begin{abstract}
:
Narrow- space surveying and mapping is of high importance for many applications, with the demand for digital 3D models in a lowcost and relatively fast way growing rapidly. This paper examines SLAM-based and image-based mapping systems for indoor mapping and focuses on the comparison of the 3D point clouds acquired from two commercial mapping systems, Leica BLK2GO and Matterport Pro2 3D (MC250). Issues that are addressed include the effect of the mapping trajectory on the produced mapping result, and the amount of mapping propagation error in narrow space surveying. The two systems are experimentally compared against both an ideal geometric target facility and in an example environment of a narrow corridor. Finally, results are given with derived metrics that can be possibly extended to several real applications.
\end{abstract}

\section{INTRODUCTION}

The last decade, technologies have emerged that simplified the data collection process towards the detailed 3D mapping of indoor spaces. Indoor narrow space surveying and mapping are of high importance for several applications. Additionally, the demand for digital 3D models of narrow spaces in a low-cost and relatively fast way is growing fast to address applications such as construction, indoor navigation, real estate management and development, emergency services, tunnel and underground mine mapping with an accuracy of few centimeters sufficed. Although this is not a new problem, it remains a complicated task due to the complexity and spatial extent of indoor structures and the lack of global references. The indoor space is fragmented to smaller spaces with diverse shapes and functions and often the layout of the indoor environment can change frequently (such as in mining tunnels) or contain moving objects (such as in public buildings), thus posing a challenge in regard to the mapping results.

Several approaches for narrow space mapping have been suggested in the literature, varying from high-accuracy techniques such as total station surveying and terrestrial laser scanning (TLS), to imaging techniques. The former provides accurate mapping results, but with a cost in labour-intensive interactive data collection and low efficiency for comprehensive indoor mapping and map/model updating. On the other hand, Simultaneous Location and Mapping (SLAM) has gained attention as a promising technology for indoor mapping. It permits the real-time creation and updating of a map while deriving location information without any prerequisite. It is usually operated with photographic sensors like cameras, ranging sensors like LiDAR or depth cameras. According to the sensors employed, the most widely used SLAM techniques are the LiDAR SLAM. Alternatively, there are image-based methodologies that use the registration of simultaneously gathered depth and color images from the respective sensors (e.g., Microsoft Kinect).

This paper attempts to assess SLAM-based and image-based mapping systems for indoor narrow space mapping through the use of different relatively low-cost system configurations. It focuses on the comparison of the 3D point clouds acquired from two commercial mapping systems and specifically the Leica BLK2GO which is SLAM-based and Matterport Pro2 3D (MC250) which is image-based and linked to SLAM technology. Some of the issues that will be addressed include the effect of the mapping trajectory on the produced mapping result, the effect of the sensor operation and the amount of mapping propagation error in narrow space surveying. Distinctive contribution of this work includes the evaluation of configurations as well as significant in situ evaluation in a metrological EDM calibration facility and a photogrammetric test field, analysis missing from prior characterization work. During the experiments, configurations with varying distance and coverage are examined. All comparisons were performed using a combination of proprietary (Geomagic, Polyworks) and open source software (CloudCompare). Whilst the obtained results are specific to the experimental configurations tested, the generality of the indoor environments and the resulting metrics can be possibly extended to several real applications. The remainder of the paper comprises three sections. In section 2 a brief description of the sensors is given and in section 3 the data collection is described. The analysis and data results are discussed in section 4 and section 5 summarises the work with concluding remarks and future directions.

\section{IMMS HANDHELD SYSTEMS}

The necessity to use known control points when collecting data is an issue of the past regarding LiDAR based systems such as with TLS. Nowadays, with Indoor Mobile Mapping Systems (IMMS), which is a relatively new and fascinating form of surveying technology, the acquisition of $3 \mathrm{D}$ and high-resolution point clouds on the move without the use of targets and control points is feasible, thus increasing productivity and opening new avenues in digitisation. In other words, an IMMS is a moving multi-sensor system which surveys the surrounding environment in a kinematic manner (e.g., Kaijaluoto et al., 2015). Currently, there are numerous commercial and other

\footnotetext{
* Corresponding author
} 
sensors presenting many instrumental details and configuration differences. Nevertheless, all systems perform the surveying task in a similar way, using a linear scanner as the measurement sensor, although some instead make use of imaging or range cameras (e.g., Khoshelham and Elberink, 2012).

From the geomatics point of view, IMMS are similar to mobile mapping systems (MMS) devised for road cadastres more than 20 years ago (e.g., Proc. of the First Int. Workshop on "Mobile Mapping Technology", Columbus, OH, USA, 1995). The revolution introduced by MMS was the direct orientation of the imaging sensors from navigation sensors, thus avoiding the classical indirect method which instead used control points and required topographic surveys. Specifically, they comprise a mapping sensor (i.e., LiDAR, active 3D imaging systems), an inertial measurement unit (IMU), a global navigation satellite system-GNSS receiver, and a time referencing unit (Sammartano and Spano, 2018). According to the acquisition mode movement, platforms can be divided on those movements that equip a vehicle (by ground, on air, or on water) or portable movements (by towing trolley, man-portable backpacks, or portable handheld devices), with or without GNSS positioning solutions (Nocerino et al., 2017).

IMMS differ from MMS in how navigation is performed. Because these are mainly for indoor use and cannot use GNSS, they tackle the problem of instantaneous navigation in a different way. The analytical methods used to solve this problem belong to the class of so-called "simultaneous localization and mapping" (SLAM) algorithms, originally developed in robotics (e.g., Cadena et al., 2016). With a SLAM algorithm it is possible to simultaneously build a map and localize the sensor within the map in real time or near real time. The integration of these diverse parameters is most often accomplished using SLAM-related algorithms. Specifically, Kalman filters are routinely used with the most popular being the Extended Kalman Filter (EKF) and Rao-Blackwellized Particle Filters (RBPF) (e.g., Carlone et al., 2011).

\subsection{Geospatial SLAM}

Geospatial SLAM is particularly effective for indoor mapping or surveys of enclosed environments. When compared to traditional survey methods for measuring indoors, (such as tape and disto or even static laser scanning) Geospatial SLAM can prove to be up to 10 times faster. As with IMMs, they comprise a LiDAR sensor and an industry grade MEMS inertial measurement unit (IMU) to estimate an initial position.
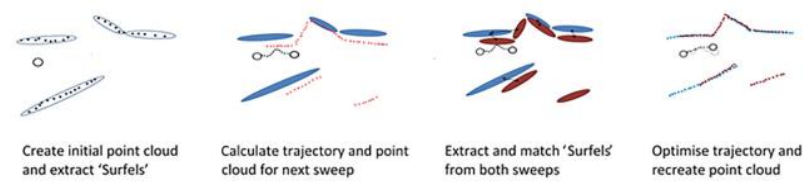

Figure 1. Basic concept of SLAM algorithm (after Reid, 2016)

The SLAM algorithms implemented in various systems are based on the same fundamental concept (Reid, 2016) but with certain modifications depending on the technology. Briefly, from the first sweep of the point cloud, sections which are called 'Surfels' are extracted to represent the unique shapes within the point cloud. The trajectory is then calculated for the next sweep of data using the IMU and 'Surfels' are extracted again in the same way. The two sets of Surfels are then used to match the point clouds together and subsequently correct and smooth the trajectory estimation. Following this iterative process, the final point cloud is recreated based on the new smoothed best estimate trajectory. To further optimise the trajectory and limit any IMU drift, a closed loop is performed such that the start and finish environments are accurately matched together (Fig. 1). Typically, SLAM works well in feature-rich environments such as buildings, however most algorithms may have difficulties in open or featureless environments such as carparks or smooth tunnels. Many SLAM algorithms only work in 2D however, it is preferable to have the robust nature of a $3 \mathrm{D}$ solution.

In this work, the BLK2GO geospatial SLAM was used (https://blk2go.com). This device uses a combination of a visual SLAM and a LiDAR SLAM capturing 420,000 points per second with a very small dual axis LiDAR enclosed in the top of the system case (dome) which performs two full revolutions in a second and rotates at $100 \mathrm{~Hz}$ on the fast axis. It is a coaxial design whereby the beam goes out and returns at the same point. There are three small but powerful panoramic cameras in the aluminium body of the instrument that are complemented by a 12-megapixel detail camera for high-contrast imagery. Specifically, the three cameras operate on a global shutter to be fast, and one camera is high resolution to tag items. A multicoloured light band encircles the device, which indicates the status of the device through colours. Prior to data collection, it requires few seconds of initialization and the device is ready when the light colour indicator changes from orange to green (Fig. 2).

The body of the BLK2GO is made from one piece of black anodized aluminium and weighs less than $800 \mathrm{~g}$ (including battery), which makes it extremely easy to carry for a long time. The battery lasts approximately 45 minutes on a full charge. The device is connected to a mobile phone where a dedicated IOS app can be downloaded and during data capture, the collected point cloud is shown in real-time. For this, a fast point cloud engine called HSPC (Hexagon Smart Point Cloud) is used in the app that enables to show the point cloud in $2 \mathrm{D}$ or $3 \mathrm{D}$ and the trajectory in real time. The data are stored in the large flash card of the device which is 512GB but when these are needed to be uploaded to the computer this is easily performed via $\mathrm{Wi}-\mathrm{Fi}$ or USB 3.0. The device's precision is specified to be better than 20mm indoors (https://blk2go.com). (a)

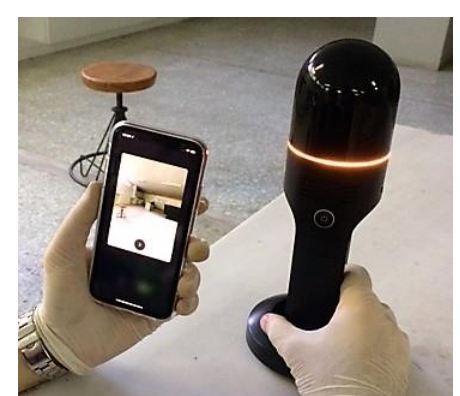

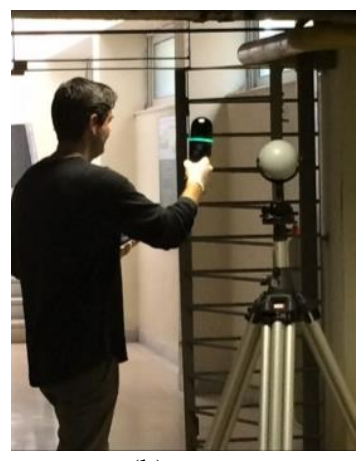

(b)
Figure 2. Use of BLK2GO during (a) initialization (b) data collection

\subsection{Matterport}

Another family of sensors linked to SLAM technology, refer to rover multi-cameras. In this work, the motorized rotating camera Matterport Pro2 3D (MC250) was used. The SLAM here is used to determine the device's position with reference to a certain scene based on RGB-D images (Endress et al., 2012).This is a combination of RBG images and depth images and uses SIFT (Scale Invariant Feature Transform) and SURF (Speed up Robust Feature) algorithms to find corresponding points. 
The system also uses a structured light (infrared) 3D sensor with capture time equal to $20 \mathrm{sec} / \mathrm{scan}$ and with a maximum operating distance of $4.5 \mathrm{~m}$. The camera of the device is formed by three different lenses, namely a photo camera, a thermal imaging infrared camera, and a depth camera. These allow to capture images in HDR and 3D data which are subsequently connected to obtain a single 3D result, in the way of polygonal mesh. In addition to the mesh, data processing through an external server, gives an equirectangular photo of $134.2 \mathrm{MP}$ (whose dimensions are 1280x1024 pixel), formed by 18 images. The cameras (in total 9, 3 for each kind of lens) are horizontally oriented and slightly inclined upwards and downwards so that the three projection centres converge in a single point. The data capture takes place through a complete rotation on the horizontal plane, generally divided into six steps whereby the frames from each step will form the final image. The typical field-of-view is on the horizontal at $360^{\circ}$ and on the vertical limited at $300^{\circ}$ (Pulcrano et al., 2019).
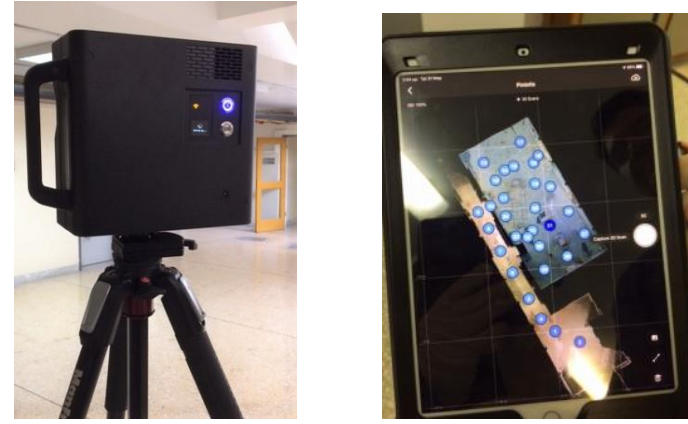

Figure 3. Matterport Pro2 3D and the display of data in the app during the acquisition

The device is managed through an IOS app called 'Matterport Capture' allowing to control the camera through a Wi-Fi connection. For every new position of the instrument, the app displays a first processing of the plan enabling to continuously verify the acquired areas and their scans in order to fill areas of shadow. The visualization during data capture is limited only to planimetry (Fig. 3). During data acquisition, there is no requirement for reference targets. Matterport Pro2 3D aligns data from two consecutive positions based on homologous points on the scene. The alignment algorithm uses elements from one image, identifying both spatial data and RGB values, so that to position the subsequent image and to correctly reconstruct its geometry without the aid of known points. The algorithm can be affected by variations in lighting conditions and displacement of objects inside the scene because of the errors produced in the different connections. The final 3D reconstruction of the space measured is the result of a combination of the real time spatial alignment algorithms and Matterport's Artificial Intelligence (AI) means, namely the Cortex deep learning neural network. Although an indoor mapping instrument, attempts have been reported for outdoor surveys (e.g., Gardin and Jimenez, 2018) with positive outcomes despite some challenges, such as low range of acquisition, the lack of overlap among the different station points and the impact of natural light.

\section{DATA SETS}

The two systems (BLK2GO and Matteport Pro2 3D) were employed to map an indoor environment of approximately 600 $\mathrm{m} 2$. The following sections describe the test environment and the datasets.

\subsection{Test Environment and Data Collection}

The test environment is part of the basement floor in the Surveying Engineering building of the National Technical University of Athens. The collected data feature a long narrow corridor of about $100 \mathrm{~m}$-long with several turns, a large room with a photogrammetric test field, and a dedicated metrological facility with an EDM calibration baseline comprised of 26 pillars and nominal length of $50 \mathrm{~m}$ (Fig. 4). During the data collection, few turns were made to enable the study of the point cloud rigidness with respect to long indoor distances. Regarding lighting, the test area is generally dark being in the basement, but small windows along the corridor still allow for natural light. Also, the test site has a flat and rather shiny floor.

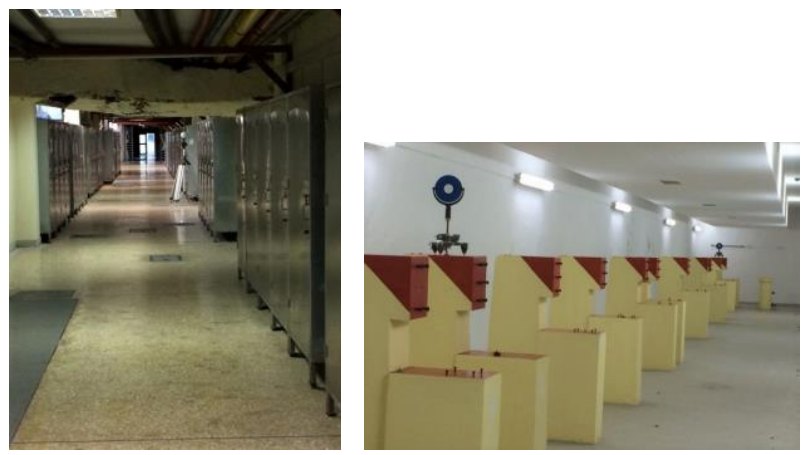

Figure 4. Views of the test environment

The use of total station surveying was implemented with the establishment of a traverse of five reference stations as control to be used for georeferencing the data sets acquired from the two devices. A spherical reflector target was positioned at each station (cf. Fig. 2) and the coordinates of the centre in all spherical targets were calculated at an accuracy of $5 \mathrm{~mm}$. In addition, the spherical targets were measured with more detail by the two different instruments to extract the point cloud for each target and model it separately. In this way, it would be possible to georeference the point clouds from both systems to the reference system of the EDM surveying.

Prior to data collection, some rules, based on the literature, were set for using both devices in the best way so that to acquire as many comparable point clouds as possible. For instance, survey resolution is related to the walking speed, the way how the handheld systems are held affects the completeness of the model, the path should be even to avoid sudden turns, large differences in the ground influence the obtainable results, and so on. In other words, trajectory and loops should be planned according to the local environment configuration. This is important because a SLAM-based system is based on the iterative alignment of extracted profiles that require featuring attributes of the space. Also, roundtrips are preferred to avoid drift error propagation or linear deviation.

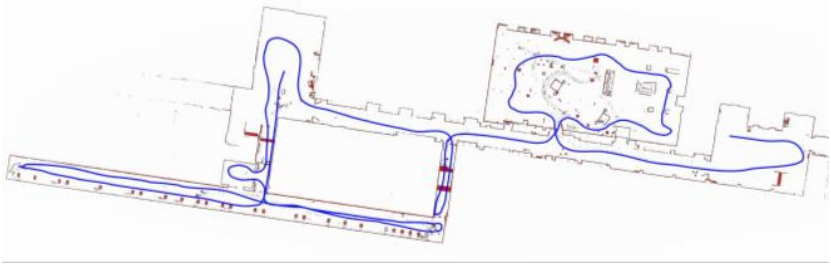

Figure 5. Test footpaths (a) path 1, open; (b) path 2, closed

For the testing of the devices, two paths were defined. The first path was an open loop starting at the right of the image (Fig. 5) 
and included elevation differences from the start point to the end point, as the level of all corridors are not the same and short staircases connect the different spaces. The duration of the walk with the BLK2GO took less than $15 \mathrm{~min}$ including the initialization of few minutes and the delay due to the inexperience of the users. The second path was a closed loop and referred only to the EDM calibration facility (Fig. 6).

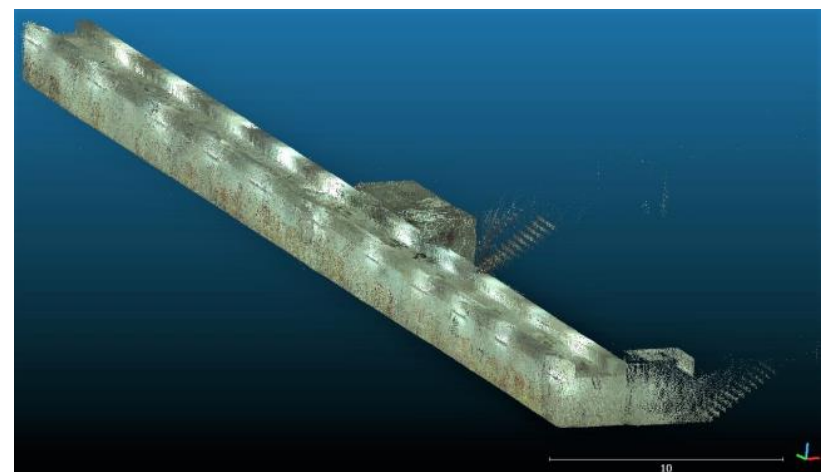

Figure 6. View of the point cloud acquired by the BLK2GO

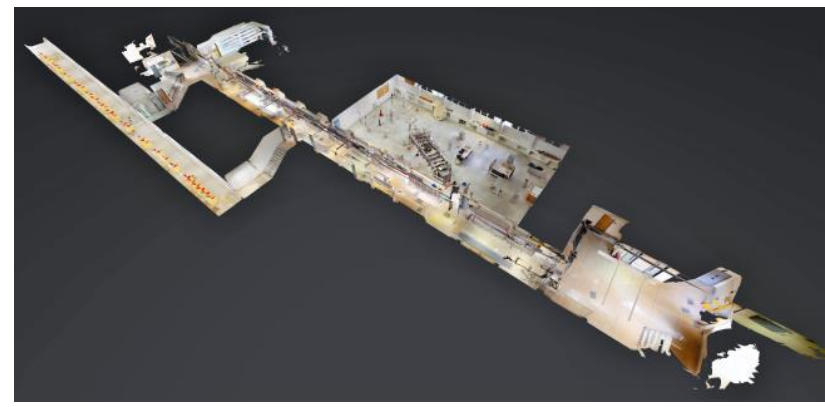

Figure 7. View of the 3D data acquired by the Matterport

The Matterport acquisition process uses a tripod mounted camera rig with three colour and three depth cameras pointing slightly up, horizontal, and slightly down. For each panorama, it rotates around the direction of gravity to 6 distinct orientations, stopping at each to acquire an HDR photo from each of the 3 RGB cameras. The three depth cameras acquire data continuously as the rig rotates, which is integrated to synthesize a 1280x1024 depth image aligned with each colour image. The result for each panorama is 18 RGB-D images with nearly coincident centres of projection at approximately the height of a human observer. The sensor was positioned at uniformly spaced intervals at approximately $2-4.5 \mathrm{~m}$ throughout the entire walkable floor plan. The data collection lasted for about $1.5 \mathrm{hrs}$ as the device was positioned on 83 stations to cover all the area (Fig. 7). Using an IOS app, the collected data were uploaded to the Matterport cloud. After few hours, the user can get the final products which is a single 3D result in the way of polygonal mesh along with a point cloud. It is emphasised here that the user cannot have any input on the derived products and the point cloud does not represent raw data in the way it results from a dedicated LiDAR-based system such as a TLS. A problem experienced during the data collection was the difficulties in alignment, especially in the metrology facility. Despite a careful evaluation of scan positions, supported by the preview of previous images through the Matterport Capture App, it was noticed that the resulted model was aligning wrong images. This was mainly due to the uniformity of the environment and lack of characteristic points of discontinuity. This lead to readjust the position of the instrument and to perform a new acquisition to help the alignment with previous acquisitions and to continue with the following ones.

\subsection{Dataset Alignment}

To analytically estimate the accuracy of positions from the two devices based on instrumental error can be problematic. The data assessment presented here is produced by comparing each resultant mapping with the 'ground truth'. The truth is provided by known distances (EDM calibration baseline and photogrammetric test field). The two examined systems provided data in different arbitrary reference systems. As it was eventually not possible to complete georeference of the two clouds, alignment was performed instead through common identified points and ICP algorithm. Therefore, both needed to be transformed in the same reference system. For this purpose, a proprietary software (Geomagic) was used. The three graphs of Fig. 8 illustrate the alignment approaches for the data. In Fig. 8a it can be seen how the two clouds are associated without any intervention. At a second approach, a rough alignment was obtained from the two clouds by rotating and translating them, and suitably defining four corresponding points in all the datasets. These points were chosen as far away from each other as possible in the whole test area: the same natural features were picked up in all the datasets. As a result, the two clouds were roughly pre-aligned (Fig. 8b).

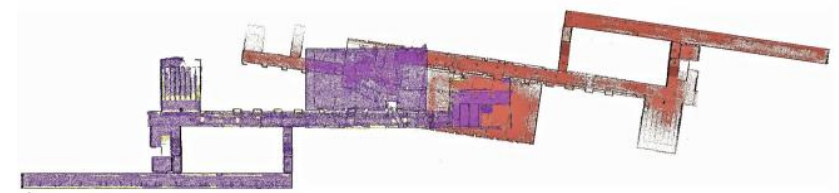

(a)

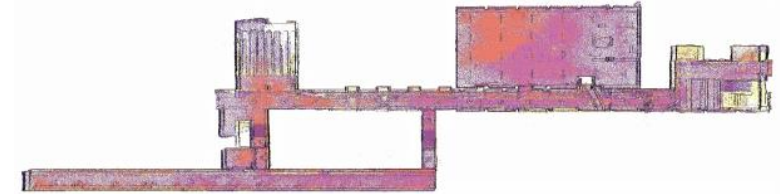

(b)

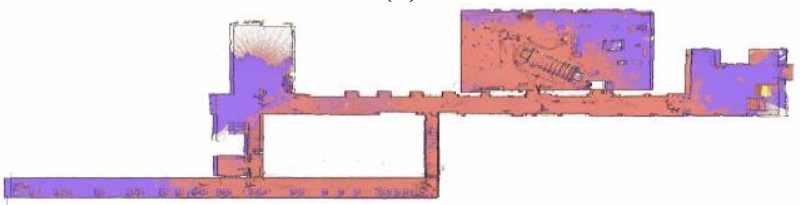

(c)

Figure 8. View of the merged point clouds: (a) without alignment; (b) manual alignment; (c) alignment with ICP

The final refinement was obtained using the ICP algorithm, again in Geomagic software. Hence, the ICP algorithm was used in a traditional manner. As is known, the ICP process randomly considers a sub-sample of the point clouds at each iteration. Due to the large point clouds considered, it was decided to perform dissemination on the cloud of the BLK2GO to have a balance between the two types of point cloud data and maintain reliability of the results. The ICP alignment used about 10,000 points with produced RMS of $10 \mathrm{~cm}$, for all data included in paths 1 and 2. A larger number of points for the ICP did not change the RMS value for the alignment. In Fig. 8c, is noticed that the alignment presents a lower discrepancy towards the central area for the Matterport cloud whilst a prominent higher discrepancy for the BLK2GO cloud is shown at the edges of the cloud.

\section{RESULTS}

The validation of the data is always essential to determine how to use them in different application fields and determine the effectiveness of the systems on different contexts. The 
validation in this work comprised geometric and qualitative comparisons.

\subsection{Geometric deviations}

To control the overall metric quality of the two IMMS clouds, their reliability was firstly considered based on their single use. The first statistical parameter that contributes to the evaluation of the overall reliability was the accuracy, so some surfaces or clouds derived from more precise measurement systems were considered as 'ground truth' to evaluate the deviation of the studied clouds by means of root mean square error (RMS). It is also necessary to consider the precision of the clouds because it is linked to the concept of repeatability of the measurements and is normally described by the standard deviation parameter. In this work the 'truth' is provided by known distances and height differences from the EDM baseline and the photogrammetric test field. Specifically, the horizontal distances and heights between the 26 survey pillars of the metrological baseline are known with an accuracy of sub-mm from extremely high accuracy sensors (i.e. laser tracker). Similarly, the distances and height differences of the targets in the photogrammetric test field are known by high precision total station (TDM 5005) surveying measurements. The above validation was performed separately for the BLK2GO and Matterport data.

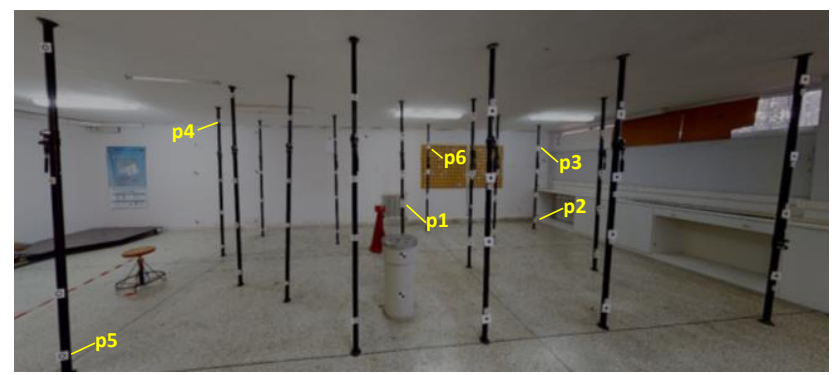

(a)

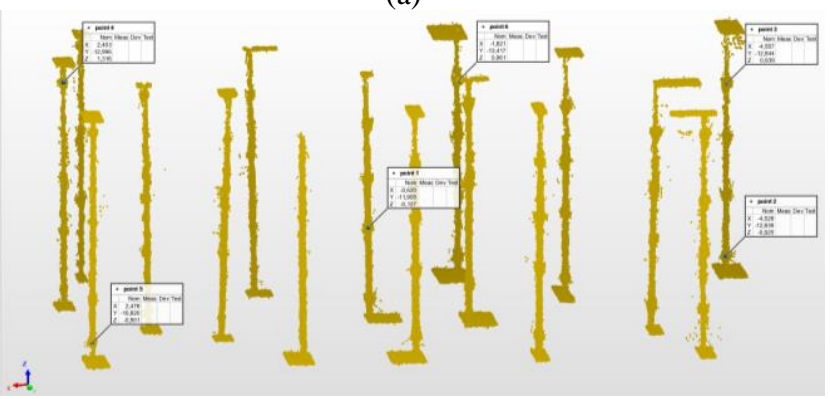

(b)

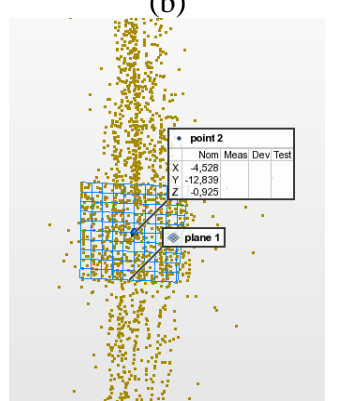

(c)

Figure 9. Photogrammetric test field: (a) image; (b) targets in BLK2GO cloud; (c) target plane fit

In Fig. 9, the slope distances between several targets, recognised in the BLK2GO cloud, were estimated. As the centre of the target was not evident in the cloud, it was decided to model each target separately by a plane fit and derive its centre geometrically (this was performed in Polyworks software). It should be emphasised that the target centre determination is affected by the number of the selected points and has a direct influence on the results.

The Matterport cloud could not depict the photogrammetric test field, as is discussed in section 4.3, and thus the specific system was not considered for this assessment. Similar procedure was followed for the EDM calibration baseline where the horizontal distances between the pillars were measured in both the BLK2GO and Matterport clouds. The mean differences from the truth with the associated standard deviations are given in Table 1 (units in metres).

Table 1. Statistics on distance comparisons

\begin{tabular}{|l|l|l|l|l|}
\hline & \multicolumn{2}{|c|}{ BLK2GO } & \multicolumn{2}{c|}{ MATTERPORT } \\
\hline & mean & std.dev. & mean & std.dev. \\
\hline EDM baseline & 0.002 & 0.013 & 0.026 & 0.027 \\
\hline Test field & 0.006 & 0.004 & - & - \\
\hline
\end{tabular}

On the contrary, when the height differences of the survey pillars were estimated on the two point clouds, it was found that the mean differences vary between $8 \mathrm{~mm}-20 \mathrm{~mm}$ for the BLK2GO cloud whilst the differences are only at the $4 \mathrm{~mm}$ $6 \mathrm{~mm}$ level for the Matterport data. This can be attributed to the fact that the quality of the Matterport data is better for defining the geometric features of the object clearer by tracing exact polylines using a skilled operator, compared to the BLK2GO data which are sparser (Fig. 10a, b). The height plane was then defined in each segment on the top of the traced polylines (Fig. 10c). It was verified also that these data have different structure and density, which in turn may lead to some problems with clouds orientation.

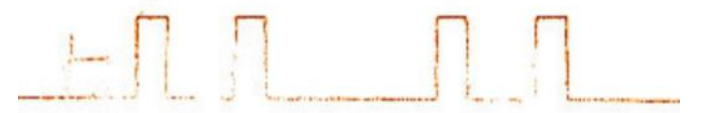

(a)

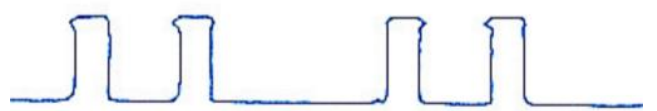

(b)

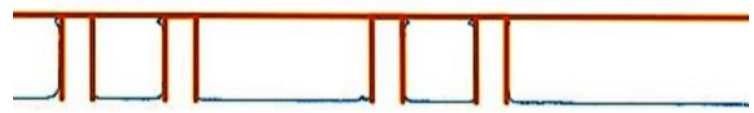

(c)

Figure 10. Segment of the survey pillars: (a) BLK2GO cloud; (b) Matterport cloud; (c) height plane

A second comparison referred to the overall metric quality of the acquired clouds based on their repeatability. This test concerns only the BLK2GO data as with the Matterport no repeated data collection was performed. Specifically, the metrology facility was acquired twice (path 1, path 2) with the BLK2GO, as described in section 3.1. Fig. 11 depicts part of the horizontal section between the two clouds where the level of accuracy, detail and the noisiness of the clouds are quite different. In fact, there is a shift of the longer path (path 1) compared to the shorter path (path 2) and the evidence of "double surfaces" produced. The SLAM system, after calculating the raw trajectories, uses an iterative ICP-like process of automatic cloud-to-cloud profile registration to generate the $3 \mathrm{D}$ cloud, and this process has always been 
controlled using loop paths and is affected by the distance of the loop. Therefore, the longer path seems to produce slightly shifted results compared to the shorter path. In this case, however, the ICP algorithm provided the short path 2 erroneous instead of path 1 mainly because of the featureless environment.

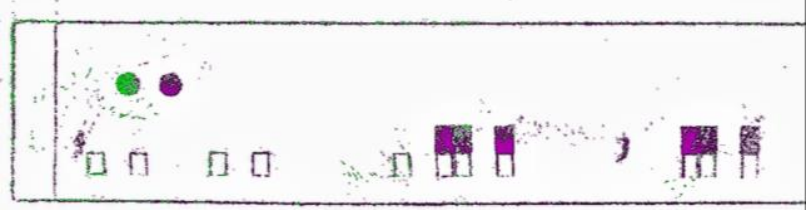

Figure 11. View of horizontal section for BLK2GO clouds (path1vs. path 2)

Whilst cloud to cloud measures are somehow local measures, i.e. are used to quantify the accuracy in the local vicinity, the global discrepancies (e.g. caused by an erroneous trajectory computation) are difficult to identify with such local methods. One possibility to detect those effects is to compare the difference between corresponding distances. For this reason, horizontal (Fig. 12) and vertical sections (Fig. 13) were made to compare the clouds from the two systems. Both figures illustrate the same part of the calibration baseline and the colour scheme corresponds to BLK2GO data with orange line and to Matterport data with blue line.
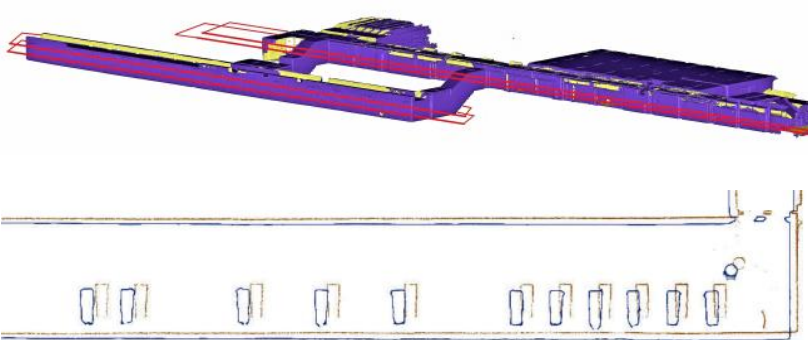

Figure 12. View of horizontal section from BLK2GO and Matterport (with scale distortion) clouds
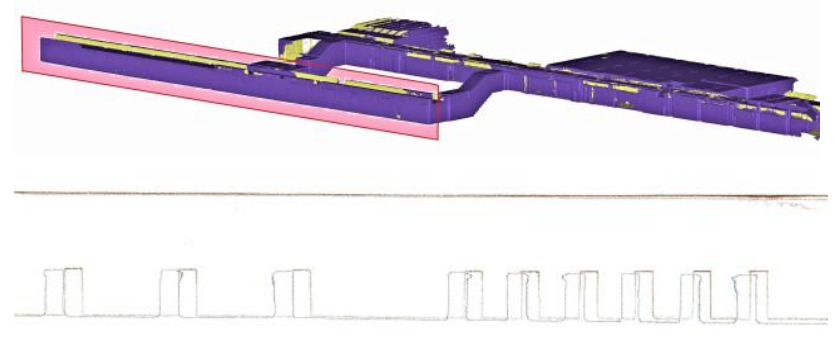

Figure 13. View of vertical section from BLK2GO and Matterport (with scale distortion) clouds

The comparison between the two clouds highlighted that the Matterport cloud has discrepancies in its total dimensions although the producer assures a high precision of 99\% (Matterport, 2019). In fact, by carrying out some sections on horizontal and vertical planes, it was shown by the analysis of the profiles of the horizontal sections, that the difference in size is probably due to a propagation of the instrument error of measurement in the scan direction. In practice, although along a not straight course, the linked acquisition led to a series of errors in the single acquisitions. In fact, once the cloud portion representing the corridor has been extracted, its length - the direction chosen for acquisition - showed an inadequate size, while its width is more consistent with truth data. Vice versa, in secondary areas, since the direction of the scan has changed, the analysis of the same data indicates that the width is outsized. For this reason, the overall effect is that the point cloud shows mistaken data in both directions. The above remarks corroborate with results from other authors (e.g., Pulcrano et al., 2019).

In general, it appears that Matterport produces models that are slightly too large, with this being most visible in the largest models. This produces significant mismatches in larger datasets when the models can no longer be fully aligned. Most significant local deviations were found in small details visible (cf. Fig. 13). There was also a minor corner rounding effect visible in models. The issue was significantly more severe when the models covered a larger area. When the measured target zone is smaller, the Matterport system produced more detailed meshes. On the other hand, with larger entities the mesh quality decreases. This is clearly visible when comparing the reconstruction of details in large areas (such as a corridor with associated rooms) and a scan of an individual small room. From the above evaluation it was evident that the Matterport data have a strong correlation with distance (i.e. scale distortion), which is in agreement with relevant literature.

\subsection{Systematic errors}

To quantify the above discrepancies, many authors in the literature make use of TLS data as reference (e.g., Shults et al., 2019; Lehtola et al., 2017). In this work, the comparison was made with known distances, as discussed in the previous section, and indicated that a scale factor needs to be calculated to account for the systematic error. The scale factor for the test data was calculated equal to 0.997 which corroborates with recent literature (e.g., Shults et al., 2019).

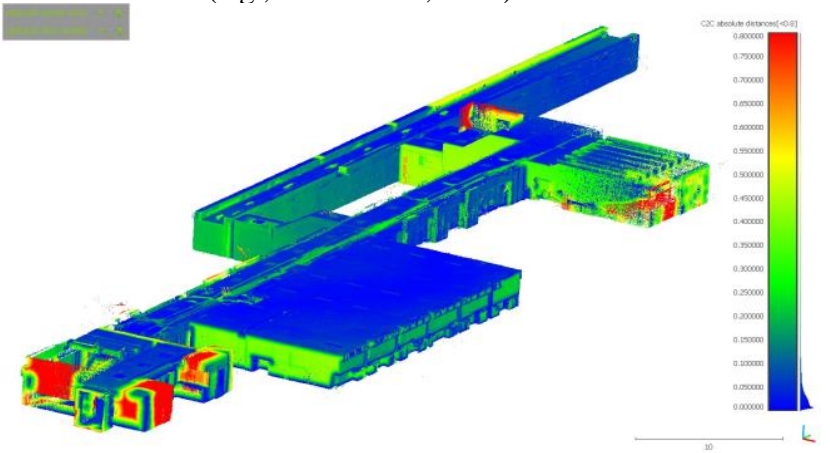

(a)

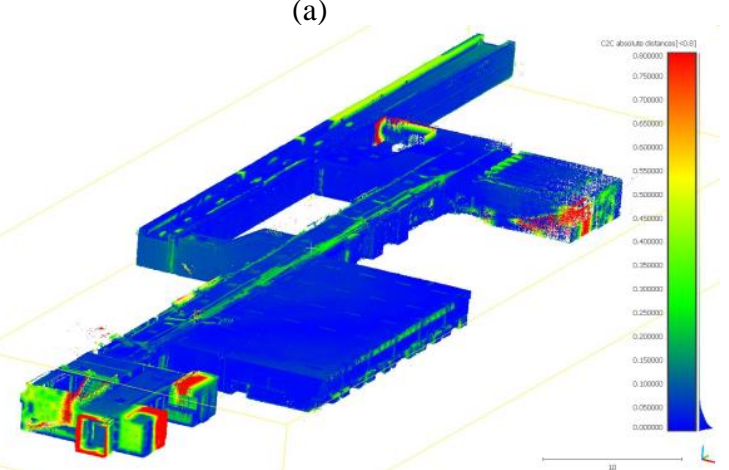

(b)

Figure 14. Aligned point clouds (a) scale distortion; (b) scale factor applied

In Fig. 14a it is shown the cloud-to-cloud comparison between the BLK2GO and Matterport data prior to scale factor correction and Fig. 14b shows the cloud-to-cloud comparison after the scale factor correction of the Matterport data. The 
above were produced in the open source Cloud Compare software. Prior the scale factor correction, the two sets of clouds have discrepancies that fluctuate between $0-0.8 \mathrm{~m}$ all around the external surfaces (mean $0.081 \mathrm{~m}$ and standard deviation $0.113 \mathrm{~m}$ ). When the scale factor is implemented, the discrepancies among those surfaces exist only for points that are farthest from the acquisition trajectory. In this case, the mean equals to $0.042 \mathrm{~m}$ and the standard deviation equals to $0.077 \mathrm{~m}$.

After the Matterport data were corrected by the scale factor, a further comparison using horizontal and vertical sections between the two types of clouds was made to examine their agreement. Fig. 15 depicts the details from the horizontal and vertical section of the calibration baseline (same part) using the two data sets. The differences between the two data types have a mean of $0.002 \mathrm{~m}$ and a standard deviation of $0.012 \mathrm{~m}$. In both graphs, the BLK2GO data are depicted in blue line and the Matterport data in orange line.

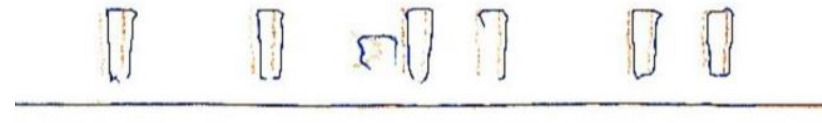

(a)

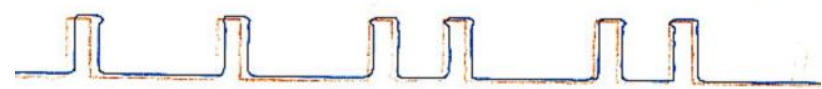

(b)

Figure 15. Details of sections with scale factor corrected: (a) horizontal; (b) vertical

\subsection{Qualitative comparison}

The qualitative evaluation concerns the completeness of the data and level of detail of the two clouds regarding certain characteristic features. Clearly, distinguishing features is strictly related to the noise level and the model resolution, both influencing the "level of detail" (LoD) of the obtained models. While the noise level mainly depends on the system (as well as on the algorithms applied in the data processing), the resolution is strongly dependent on the acquisition speed. Perhaps, an improved working mode to enhance the obtainable LoD can simply be to slow the walking speed in the proximity of objects of special interest.

Several features in both clouds were evaluated and compared regarding their recognizability. Specifically, spherical targets, survey pillars in the metrology facility, the photogrammetric test site and architectural details such as metallic objects where chosen to examine if are recognized (or not). The following figures give examples of the above. From Fig. 16a, b it is seen that the BLK cloud appears to be composed of points formations resembling to "lines" rather than points in various directions. The survey pillars are formed with their correct dimensions but regarding the spherical target, this is only possible to "glimpse" it (right pillar) but is not feasible to detect the $15 \mathrm{~cm}$ diameter sphere. The Matterport cloud depicts the same objects but in a sharper form and the spherical target is better formed but still not adequate to model it correctly.
Clearly, the LoD from both clouds is not adequate to recognize the objects and precisely compute the sphere centre.

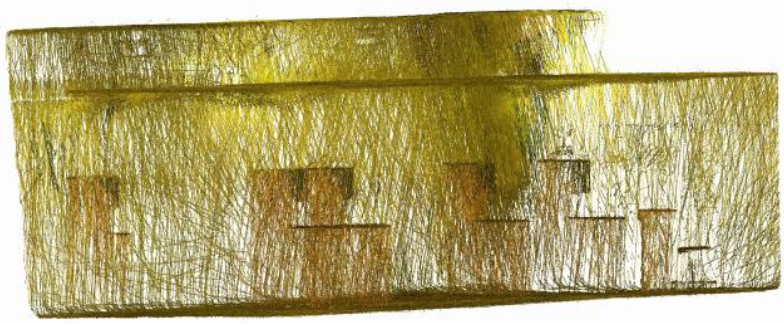

(a)

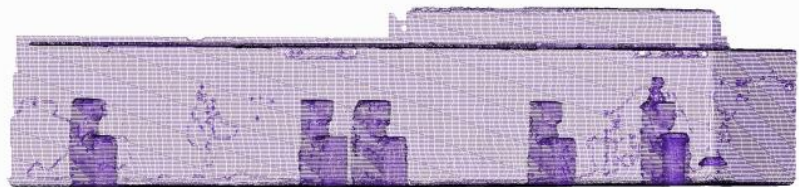

(b)

Figure 16. Point cloud of survey pillars (a) BLK2GO (b) Matterport

Figures $17 \mathrm{a}$ and $\mathrm{b}$ show the photogrammetric test field that is comprised from special pillars with targets attached on them. The pillars are placed in different horizontal levels and the attached targets are set in various vertical levels to accommodate photogrammetric checks. The BLK cloud depicts adequately the pillars with the attached targets but there are noticeable distortions in the pillars which do not exist. The Matterport cloud has extremely low density regarding the specific objects and was therefore not possible use the information further. However, the two survey pillars can be seen clearly in the scene.

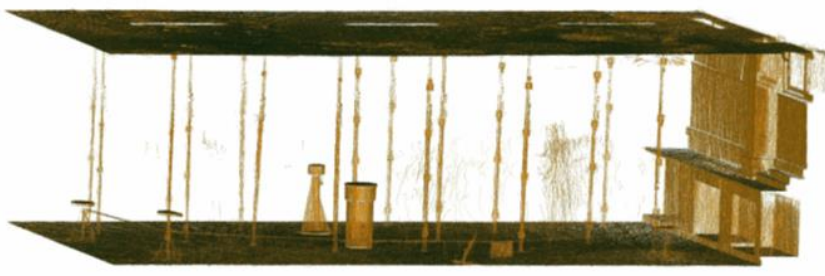

(a)

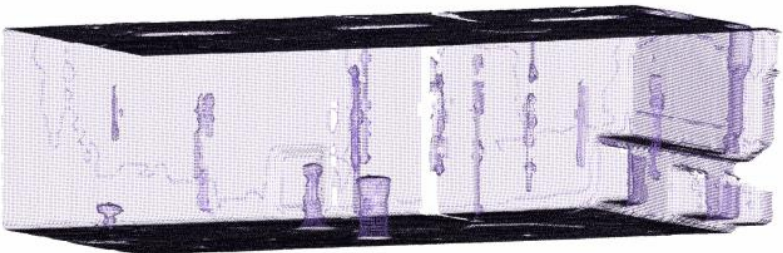

(b)

Figure 17. Point cloud of the photogrammetric test field: (a) BLK2GO; (b) Matterport

To assess the usability of the data in an urban/architectural environment, some comparisons were made with respect of not only recognizing volumes, but also identify typical architectural details such as doors, windows, and so on. Here, the recognizability of details from the two systems can be easily appreciated. The specific objects shown in Fig. 18a, b refer to metallic closets where students keep safely the surveying equipment. Due to use of LiDAR, reflective materials like glass and mirrors, may cause problems by reporting wrong range data 
to the reflecting obstacle, thus potentially causing a collision. It can also get wrong reflected points, which will cause errors in the maps generated by a SLAM algorithm (Zhao et al., 2020). Both systems provide data with correct geometric characteristics of the objects (shape and size). In Fig. 18a it is seen that the BLK2GO data present larger gaps due to reflection compared to Matterport data (Fig. 18b) but the latter shows sparsity of data at the top of the closets.

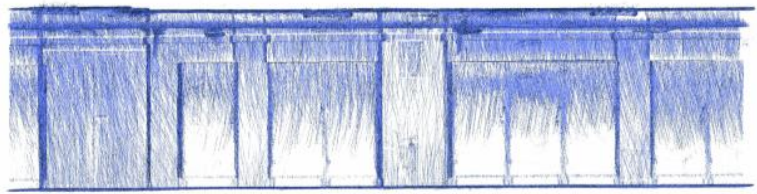

(a)

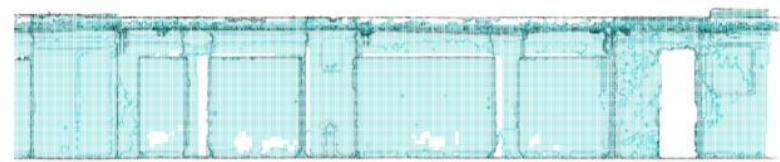

(b)

Figure 18. Point cloud of architectural details: (a) BLK2GO; (b) Matterport

It was noticed in both datasets, that the resolution varies greatly on the surfaces, being higher along the scanned profiles and lower between one profile and the next. Thus, the results cannot be generalized. The BLK2GO clouds present smaller differences and more complete model with respect to reality whilst Matterport provides data with higher intensity enabling the identification of artificial features.

\section{CONCLUDING REMARKS}

The mobile scanner technology is developing rapidly to reach more and more high precision results in short time and clearly, the future direction of survey technologies will be to transfer from static instruments to mobile ones in many application fields. In fact, the development of a hybrid and versatile solution composed by different types of sensors integrated in the same shell is seen as a solution that may fulfil the needs of challenging environments that need mapping. The difficulties of this technology relate to the demand of alignment verifications for loop configuration and closure.

In this work, two different commercial systems for indoor mapping were examined. Clearly, is complicated to compare different systems and technology, considering that hardware/software configurations can dramatically change from one to another and, above all, that the results are strongly influenced by the adeptness of the software.

A comparative procedure was followed considering the point clouds obtained by the SLAM BLK2GO and the Matterport Pro2 3D systems in an environment where it was possible to reproduce real, variable conditions as well as provide control that served as the 'ground truth'. Currently, the BLK-derived models can be considered to correspond to accuracies that represent scales of 1:100 or 1:200. For scales 1:50 or above, static systems such as total station surveying, and photogrammetry are still required. Also, a problem in SLAM technology is the lack of radiometric information that would seem to be the next step in the updated systems. On the other hand, Matterport Pro2 3D is a particularly good alternative with advantages including the management of survey campaigns, the speed of data acquisition without the aid of targets, the quality of RGB-D images of even complex spaces. Despite the scaling errors detected, when special measures are taken by decreasing the distance between station points of the instrument and making acquisition following a closed course, the metric error propagation in data is reduced.

\section{ACKNOWLEDGEMENTS}

The authors acknowledge the support of Metrica S.A. Greece for providing the equipment BLK2GO and Matteport Pro2 3D used in this work, especially to A. Skassi and A. Sardelianos.

\section{REFERENCES}

Cadena, C., Carlone, L., Carrillo, H., Latif, Y., Scaramuzza, D. Neira, J., Reid, I., Leonard, J.J., 2016. Past, present, and future of simultaneous localization and mapping: Toward the robust-perception age. IEEE Trans. on Robotics, 32(6), 1309-1332.

Carlone, L., Kaouk Ng, M., Du, J., Bona, B., Indri, M., 2011. Simultaneous Localization and Mapping Using RaoBlackwellized Particle Filters in Multi Robot Systems. J Intell Robot Syst, 63, 283-307.

Endress, F., Hess, J., Engelhard, N., Sturm, J., Cremers, D., Burgard, W., 2012. An Evaluation of the RGB-D SLAM System. Int. Conf. on Robotics and Automation, 1691-1696.

Gärdin, D., Jimenez, A., 2018. Optical methods for 3D reconstruction of railway bridges - Infrared scanning, Close range photogrammetry and Terrestrial laser scanning. MSc Thesis, Dep. of Civil, Env. \& Natural Resources Eng., Luleå Univ. of Technology, Sweden, http://ltu.divaportal.org/

Kaijaluoto, R., Kukko, A., Hyyppä, J., 2015. Precise indoor localization for mobile laser scanner. Int. Arch. Photogramm. Remote Sens. Spat. Inf. Sci., XL-4/W5, 1-6.

Khoshelham, K., Elberink, S.O., 2012. Accuracy and resolution of Kinect depth data for indoor mapping applications. Sensors, 12, 1437-1454.

Lehtola, V.V., Kaartinen, H., Nuchter, A., Kaijaluoto, R., Kukko, A., Litkey, P., Honkavaara, E., Rosnell, T., Vaaja, M.T., Virtanen, J.P., Kurkela, M., El Issaoui, A., Zhu, L., Jaakkola, A., Hyyppa, J., 2017. Comparison of the selected state-of-the-art 3D indoor scanning and point cloud generation methods. Remote Sensing, 9(8), 796.

Matterport, 2019. Matterport Pro 3D Camera specifications. https://matterport.com/pro2-3d-camera/ (Accessed: 3/2020).

Nocerino, E., Menna, F., Remondino, F., Toschi, I., RodríguezGonzálvez, P., 2017. Investigation of indoor and outdoor performance of two portable mobile mapping systems. SPIE 2017 (p. 103320I). https://doi.org/10.1117/12. 2270761.

Pulcrano, M., Scandurra, S., Minin, G., di Luggo, A., 2019. 3D cameras acquisitions for the documentation of cultural heritage. Int. Arch. Photogramm. Remote Sens. Spatial Inf. Sci., XLII-2/W9, 639-646.

Reid, M., 2016. What is Geospatial SLAM? LiDAR Magazine, 6(4), Spatial Media.

Sammartano, G., Spanò, A., 2018. Point clouds by SLAMbased mobile mapping systems: accuracy and geometric content validation in multisensor survey and stand-alone acquisition. Applied Geomatics, 10, 317-339.

Shults, R., Levin, E., Habibi, S., Shenoy, O., Honcheruk, T., Hart, Z., An, R., 2019. Capability of Matterport 3D camera for industrial archaeology sites inventory. Int. Arch. Photogramm. Remote Sens. Spatial Inf. Sci., XLII-2/W11.

Zhao, X., Yang, Z., Schwertfeger, S., 2020. Mapping with Reflection - Detection and Utilization of Reflection in 3D Lidar Scans. IEEE Conference on Robotics and Automation (ICRA) 2020, Paris, France. 